\title{
Few-nucleon systems at MAMI and beyond
}

\author{
W.U. Boeglin ${ }^{\mathrm{a}}$ \\ Physics Department, Florida International University, University Park, Miami, FL 33199, USA \\ / \\ Published online: 10 May 2006 - C Società Italiana di Fisica / Springer-Verlag 2006
}

\begin{abstract}
Few-body systems provide a testing ground for models of the NN interaction, reaction mechanisms and for models of nuclei. An overview of results of coincidence experiments on the deuteron, ${ }^{3} \mathrm{He}$ and ${ }^{4} \mathrm{He}$ obtained in the last 20 years at MAMI and at other facilities, covering a wide range of momentum and energy transfers, is presented.
\end{abstract}

PACS. 25.10. + s Nuclear reactions involving few nucleon systems - 25.30.-c Lepton-induced reactions 25.30.Fj Inelastic electron scattering to continuum

\section{Introduction}

Few-body systems are ideal to investigate fundamental problems in nuclear physics such as the ground state and continuum wave functions, the importance of correlations and the structure of the electromagnetic current operator. In addition, interaction effects such as meson exchange currents (MEC), and isobar configurations (IC) can be studied. At large momentum transfers, one hopes to be able to explore the transition from the regime where observables are best described by nucleon/meson degrees of freedom to the regime where quark/gluon degrees of freedom are the most efficient, in describing the interaction.

The few-body systems presented here consist of the deuteron and the ${ }^{3} \mathrm{He}$ and ${ }^{4} \mathrm{He}$ nuclei. They range from a loosely bound system, such as the deuteron, to a very tightly bound one such as ${ }^{4} \mathrm{He}$. In contrast to complex nuclei, the structure of few-body systems can nowadays be calculated with high precision using realistic nucleonnucleon interaction potentials. Very successful methods for the calculation of bound and continuum state wave functions include the solution of Faddeev-Yakubovsky [1] equations, Variational Monte Carlo [2], and other MonteCarlo-based calculations [3,4].

Many laboratories have contributed to the study of few-body systems in the last twenty years (in alphabetical order): ALS (Saclay, France), ELSA (Bonn, Germany), Jefferson Lab or JLAB (Newport News, VA, USA), MAMI (Mainz, Germany), MIT-Bates (Middleton, USA), NIKHEF (Amsterdam, The Netherlands), and SLAC (Stanford, CA, USA). Recent electron accelerators such as the Mainz Microtron and Jefferson Lab (CEBAF) provide very high-intensity, continuous wave (CW) beams. These have made coincidence experiments possible that

\footnotetext{
${ }^{a}$ e-mail: boeglinw@fiu.edu
}

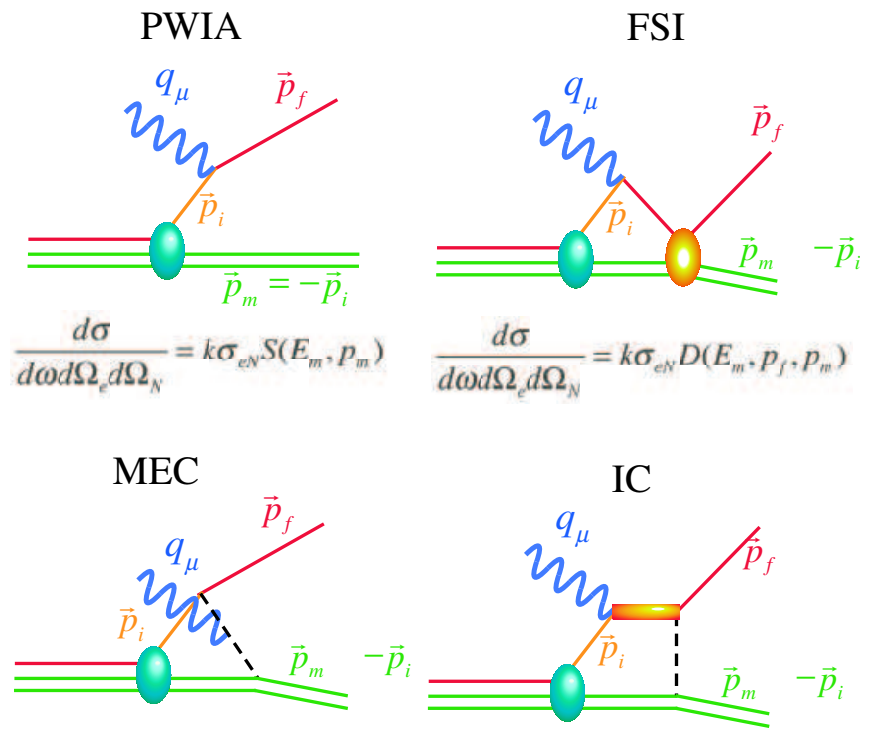

Fig. 1. Various reaction mechanisms contributing to the $\left(\mathrm{e}, \mathrm{e}^{\prime} \mathrm{N}\right)$ reaction. Plane-wave impulse approximation (PWIA), distorted-wave impulse approximation (FSI), meson exchange currents (MEC) and isobar configurations (IC).

explore new, previously inaccessible kinematical regions with very high statistical precision. I will therefore focus on the study of few-body systems using knock-out reactions such as $\left(\mathrm{e}, \mathrm{e}^{\prime} \mathrm{N}\right)$ and $\left(\mathrm{e}, \mathrm{e}^{\prime} \mathrm{NN}\right)$.

\section{Short overview of the $\left(e, e^{\prime} N\right)$ reaction}

Treating the incoming and scattered electrons as plane waves, and applying the one-photon exchange approximation, the $\left(\mathrm{e}, \mathrm{e}^{\prime} \mathrm{p}\right)$ reaction can be viewed schematically as 


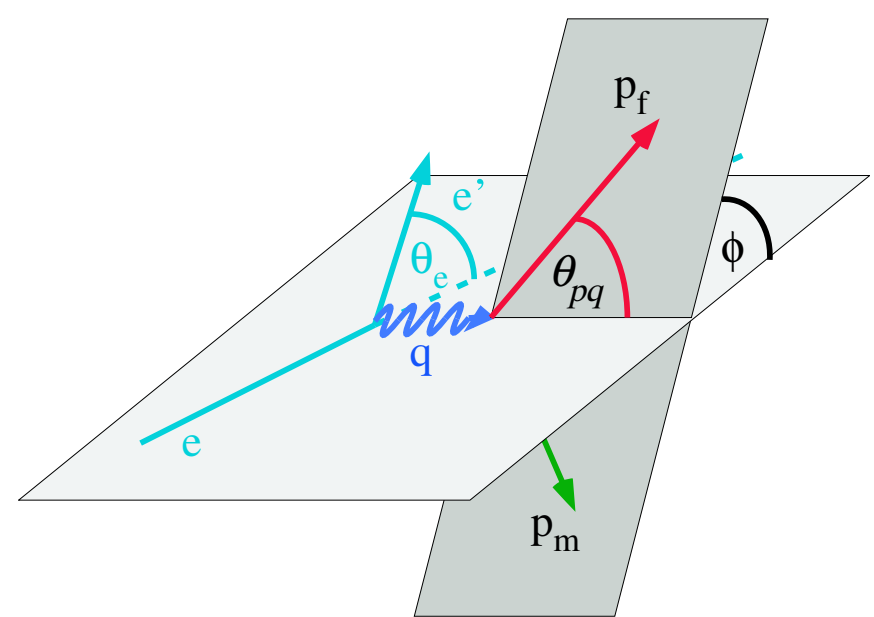

Fig. 2. Geometry and kinematical variables for the $\left(e, e^{\prime} N\right)$ reaction.

shown in fig. 1. In the case of the Plane-Wave Impulse Approximation (PWIA) the virtual photon is absorbed by a bound nucleon having a certain initial momentum $\boldsymbol{p}_{i}$. The struck proton subsequently leaves the nucleus with a final momentum $\boldsymbol{p}_{f}$. The residual system may remain in its ground or in an excited state and has a recoil momentum $\boldsymbol{p}_{m}$. In the following, the term missing momentum will be used synonymously with recoil momentum. Within PWIA the following relation between initial and missing momentum is valid: $\boldsymbol{p}_{m}=-\boldsymbol{p}_{i}$. The transferred energy $\omega$ is divided between the kinetic energy of the ejected nucleon, its separation energy, and the kinetic and, possibly, excitation energy of the residual system. The missing momentum $p_{m}$ and missing energy $E_{m}$ are defined as follows:

$$
\begin{aligned}
& \text { Momentum conservation : } \boldsymbol{q}=\boldsymbol{p}_{f}+\boldsymbol{p}_{m}, \\
& \text { Energy conservation : } \quad E_{m}=\omega-T_{p}-T_{r} .
\end{aligned}
$$

Here $T_{p}$ is the kinetic energy of the ejected nucleon, and $T_{r}$ is the kinetic energy of the recoiling system, calculated from $\boldsymbol{p}_{m}$ under the assumption that the undetected (A$1)$-system remains in its ground state.

Figure 2 shows the electron scattering plane, defined by the incoming and scattered electron momenta, and the reaction plane, defined by the final nucleon momentum and the momentum transfer. The cross section in the one photon exchange limit can be written as $[5,6,7]$

$$
\begin{aligned}
\frac{\mathrm{d}^{5} \sigma}{\mathrm{d} \omega \mathrm{d} \Omega_{e} \mathrm{~d} \Omega_{p}}= & \sigma_{M o t t}\left(v_{L} R_{L}+v_{T} R_{T}+\right. \\
& \left.+v_{L T} R_{L T} \cos \phi+v_{T T} R_{T T} \cos 2 \phi\right)
\end{aligned}
$$

where $R_{i}$ are the response functions containing matrix elements of the charge and current operators. These, in turn, provide the nuclear structure information. The $v_{i}$ are kinematical factors depending on the electron kinematics only, and $\sigma_{M o t t}$ is the Mott cross section describing the scattering of relativistic electrons by a point charge.

If one neglects the interaction of the outgoing nucleon with the recoiling system, one obtains the plane-wave impulse approximation (PWIA) which permits a factoriza-

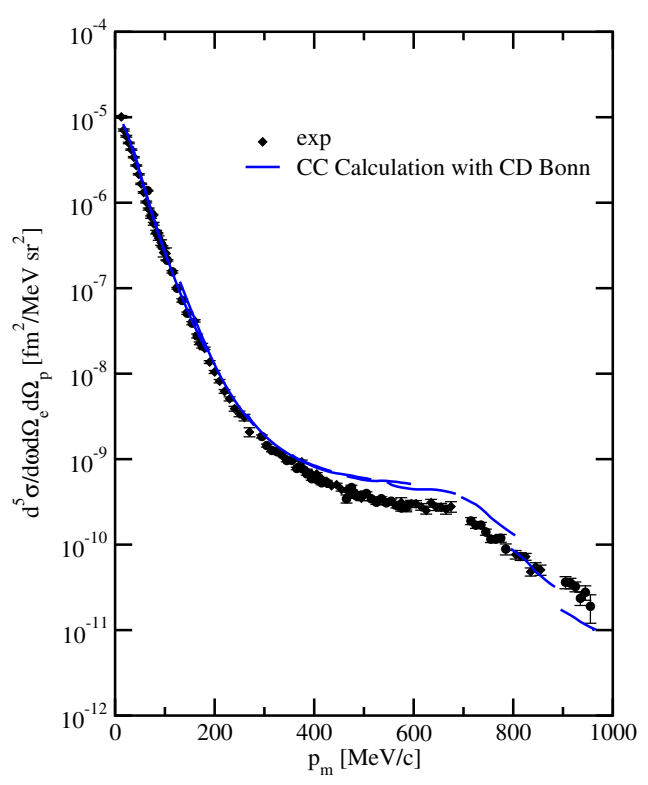

Fig. 3. D(e, e'p)n cross section measured at MAMI [11] compared to calculations by H. Arenhövel [13].

tion of the $\left(e, e^{\prime} \mathrm{N}\right)$ cross section into an elementary (offshell) electron nucleon cross section [8] and the spectral function describing the probability of finding a nucleon with a given initial momentum and missing energy. Integrating the spectral function over the missing energy leads to the momentum distribution. Final state interactions (FSI), MEC, and IC remove this simple relation and therefore $\boldsymbol{p}_{i} \neq-\boldsymbol{p}_{m}$ (fig. 1).

\section{Studies of the deuteron}

Early $\left(e, e^{\prime} p\right)$ experiments were limited in luminosity by the duty factor of the available electron accelerators. Cross sections could be measured for large missing momenta $\left(p_{m} \approx 0.5(\mathrm{GeV} / c)\right)$ only at relatively small momentum transfer $\left(Q^{2} \approx 0.1(\mathrm{GeV} / c)^{2}\right)$ or for large $Q^{2}$ only at relatively small $\left(p_{m}<0.2(\mathrm{GeV} / c)\right)$.

Experiments have been carried out at all facilities mentioned above. More recent experiments, carried out in the last ten years, benefited from the availability of high duty cycle beams at Jefferson Lab, MAMI, NIKHEF(AmPS), and at MIT-Bates(SHR). In general, the various experiments can be separated into those that explored the $\mathrm{D}\left(\mathrm{e}, \mathrm{e}^{\prime} \mathrm{p}\right) \mathrm{n}$ cross section over a large range of missing momenta and those that extracted individual response functions.

\subsection{Cross section measurements at low $Q^{2}$}

These experiments explored the $\mathrm{D}\left(\mathrm{e}, \mathrm{e}^{\prime} \mathrm{p}\right) \mathrm{n}$ cross section over a wide range of missing momenta at small to medium 


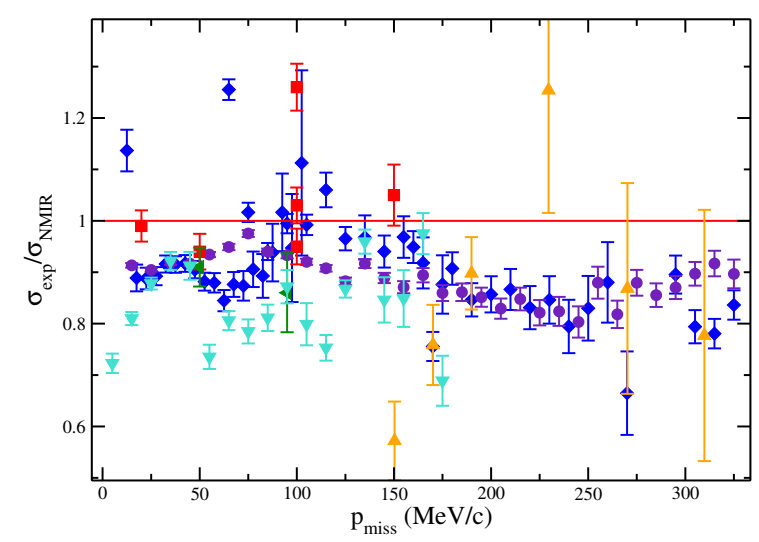

Fig. 4. Ratio of experiment to theory for low missing momenta. Data points: blue diamonds [11], red squares [14], violet circles [12], turquoise triangles down [9], green triangles left [15], orange triangles up [16].

momentum transfers $[9,10,11,12]$. The focus of these measurements was the exploration of the momentum distribution within the plane-wave impulse approximation. It has been found, however, that with increasing recoil momentum FSI and, related to the corresponding energy transfer, MEC and IC contributions increase dramatically. Figure 3 shows the $\mathrm{D}\left(\mathrm{e}, \mathrm{e}^{\prime} \mathrm{p}\right) \mathrm{n}$ cross section measured at MAMI [11] and H. Arenhövel's calculation that includes FSI, MEC, and IC [13]. One can see that the cross section is well reproduced up to $p_{m}=350 \mathrm{MeV} / c$. At higher $p_{m}$ there are significant discrepancies between experiment and theory. This occurs in a kinematical region where large virtual delta excitation contributions are expected.

Since many experiments have measured the $\mathrm{D}\left(\mathrm{e}, \mathrm{e}^{\prime} \mathrm{p}\right) \mathrm{n}$ cross section at missing momenta below $300 \mathrm{MeV} / c$ it is interesting to compare how well these results agree with each other in order to learn how accurately the $\mathrm{D}\left(\mathrm{e}, \mathrm{e}^{\prime} \mathrm{p}\right) \mathrm{n}$ reaction is known experimentally. As the various experiments have been carried out at different kinematical settings, I used Arenhövels calculation [13] as a reference to take into account FSI. MEC and IC are also included, however they tend to contribute less than FSI. The result is shown in fig. 4 where the ratio between the experimental and the theoretical cross sections is shown. It is interesting to note that the various experiments agree quite well among each other, while the experimental cross sections seem to be systematically smaller than the calculated cross sections by about $10 \%$. The reason for this discrepancy needs further investigation but one has to keep in mind that most experiments quote systematic errors around $5 \%$.

\subsection{Structure function separations}

Experiments to extract various response functions of the $\mathrm{D}\left(\mathrm{e}, \mathrm{e}^{\prime} \mathrm{p}\right) \mathrm{n}$ reaction have been carried out at most electron accelerators. The major results of these experiments will be presented below. In general, all published re-
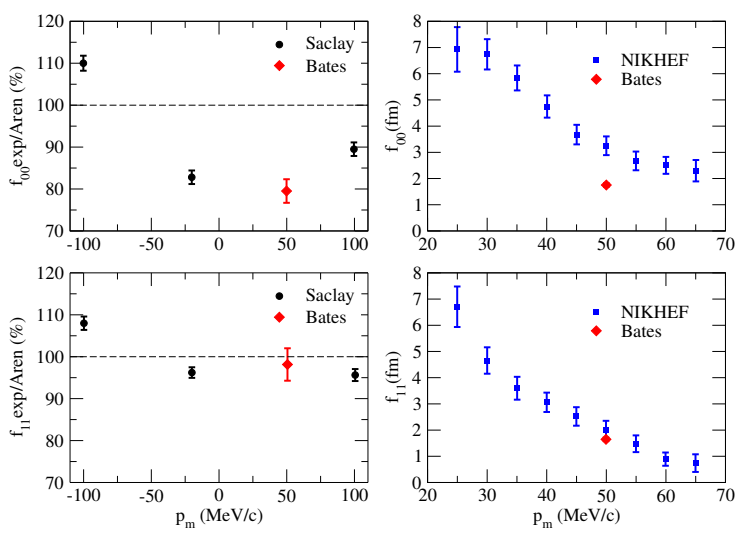

Fig. 5. Comparison of $R_{L}\left(f_{00}\right), R_{T}\left(f_{11}\right)$ measurements to one another and theory. Left: Ratio (in \%) of the experimental response functions from Saclay [14] and Bates [15] to the calculation by H. Arenhövel [19], [20]. Right: Response functions from NIKHEF $[17,18]$ and Bates [15].

sponse function separations have been limited to missing momenta below $200 \mathrm{MeV} / c . R_{L}$ and $R_{T}$ have been determined at NIKHEF $[17,18]$, Saclay [14], and at MITBates [15].

Figure 5 shows a comparison of the various results for overlapping kinematics. The left panel shows the ratio to H. Arenhövels calculation $[19,20]$. The longitudinal response has been found to deviate up to $20 \%$ from the calculation depending on the missing momentum. The transverse response, depending on the missing momentum, deviates up to $10 \%$. For missing momenta below $50 \mathrm{MeV} / c$ the longitudinal response has been found to be about $20 \%$ smaller than the calculation in both, the Saclay and the Bates experiments. The transverse response has been found to be about $4 \%$ smaller than the calculation for the Saclay data and in agreement (within the error bars) for the Bates experiment. In contrast the longitudinal response found at NIKHEF is much larger than the MIT-Bates result, while the transverse responses are in agreement.

At MAMI $R_{L}$ and $R_{T}$ have been extracted for missing momenta up to $350 \mathrm{MeV} / c$ [21]. An example of the result of this experiment is shown in fig. 6 .

Unfortunately at small recoil momenta problems with the target lead to uncertainties in determining the absolute cross section making it impossible to compare the results to the low $p_{m}$ results discussed above.

To summarize, the experimental knowledge of $R_{L}$ and $R_{T}$ is limited to low recoil momenta and there are disagreements among different experiments and also when compared to modern calculations. Currently there exists no experimental program to address these problems.

The response function $R_{L T}$ is sensitive to relativistic contributions to the electromagnetic current operator [22] and FSI and has the advantage that it is particularly easy to extract. This is due to the fact that the electron kinematics are fixed and only the proton scattering 

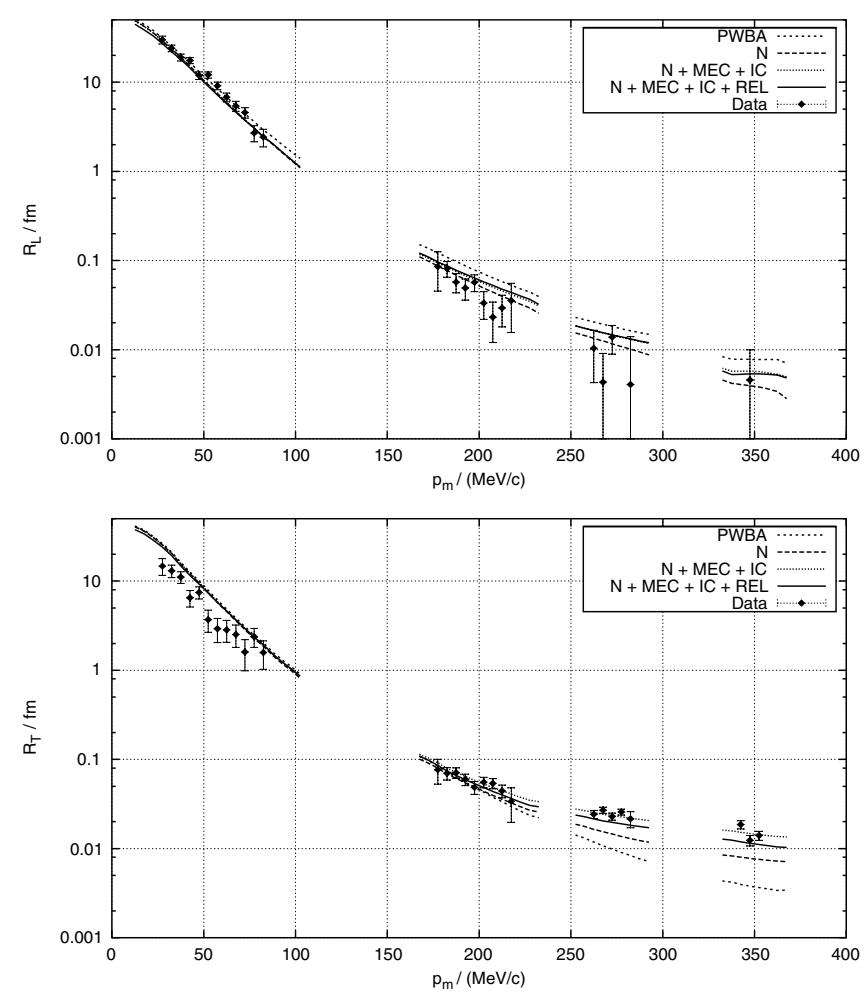

Fig. 6. Result of a $R_{L}-R_{T}$ separation at MAMI [21] for missing momenta up to $350 \mathrm{MeV} / c$ at a momentum transfer of $450 \mathrm{MeV} / c$. Calculations are by H. Arenhövel [20] (PWBA: no FSI but scattering off the neutron and observing the recoiling proton has been included).
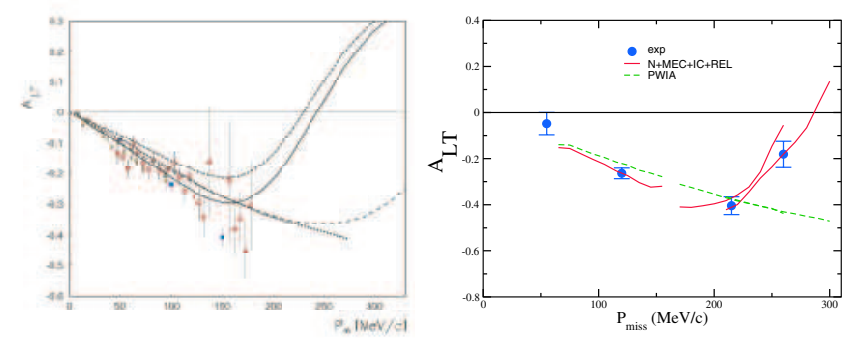

Fig. 7. Left: an overview of measurements of $A_{L T}$ from various experiments [25] where $0.15 \leq Q^{2} \leq 0.22(\mathrm{GeV} / c)^{2}$. Calculations are from H. Arenhövel et al. [26] (dash-dot: $\mathrm{N}+\mathrm{MEC}+\mathrm{IC}$, solid: $\mathrm{N}+\mathrm{MEC}+\mathrm{IC}+\mathrm{RC}$ ) and from E. Hummel et al. [27] (dashed: PWBA), the dotted curve corresponds to PWIA $\left(\sigma_{c c 1}\right) .0 .15$ to 0.22 . Right: Determination of $A_{L T}$ at MAMI for $Q^{2}=0.33(\mathrm{GeV} / c)^{2}$ [23]. The calculation is by H. Arenhövel [20] including FSI, MEC, IC and Relativistic corrections (RC).

angle is changed (in the electron scattering plane) in such a way, that the reaction plane varies between $\phi=0^{\circ}$ and $\phi=180^{\circ}$ (see fig. 2). The cross section difference obtained from these two measurements is then proportional to $R_{L T}$. A quantity closely related to $R_{L T}$ is the "left-right" asymmetry

$$
A_{L T}=\frac{\sigma_{180^{\circ}}-\sigma_{0^{\circ}}}{\sigma_{180^{\circ}}+\sigma_{0^{\circ}}}
$$

and has the additional advantage that the absolute cross section normalization cancels in the ratio. An overview of experimental results is shown in fig. 7 together with the result of a determination of $A_{L T}$ at MAMI [23]. Other recent $A_{L T}$ measurements have been published in references [16] and [24].

A determination of $R_{T T}$ requires proton detection out of the electron scattering plane. This has been achieved at MIT-Bates using the Out-Of-Plane (OOPS) spectrometer [24] system and at NIKHEF [28] using the HADRON detectors. For an overview of results see [25].

An additional response function, $R_{L T}^{\prime}$, can be obtained using out-of-plane detection of the proton and measuring the helicity dependence of the cross section with polarized electrons. This has been carried out at MIT-Bates [29] using OOPS.

\subsection{Cross section measurements at high $\mathbf{Q}^{2}$}

$\mathrm{D}\left(\mathrm{e}, \mathrm{e}^{\prime} \mathrm{p}\right) \mathrm{n}$ cross sections have been obtained at SLAC for high $Q^{2}$ but low recoil momenta $\left(p_{m}<0.2 \mathrm{GeV} / c\right)$ [30]. Recently, experiments have been carried out at Jefferson Lab in Hall A (experiment E01-020) as well as in Hall B using CLAS (experiment E94-019). The goal of the Hall A experiment is to test the Generalized Eikonal Approximation (GEA) description of FSI [31] in the D(e, $\left.e^{\prime} p\right) n$ reaction while the goal of the Hall $\mathrm{B}$ experiment is to use the GEA description of the $\mathrm{D}\left(\mathrm{e}, \mathrm{e}^{\prime} \mathrm{p}\right) \mathrm{n}$ reaction in the search for evidence of color transparency. Within the GEA, FSI are described by a series of small-angle scatterings of the outgoing nucleon. This approximation, which is typically valid for nucleon energies of $1 \mathrm{GeV}$ and above, has been successfully applied in high-energy nucleon scattering. However it has never been tested for the $\mathrm{D}\left(\mathrm{e}, \mathrm{e}^{\prime} \mathrm{p}\right) \mathrm{n}$ reaction. Another goal of the Hall A experiment is the determination $R_{L T}$ for missing momenta up to $0.5 \mathrm{GeV} / c$ where relativistic effects are expected to be very large and $R_{L T}$ is sensitive to details of the current operator.

The GEA predicts a characteristic dependence of the strength of FSI on the angle of the recoiling neutron with respect to the momentum transfer and on the value of the missing momentum. For angles around $80^{\circ}$ FSI effects are predicted to be maximal. For $p_{m}=0.2 \mathrm{GeV} / c$ a reduction of the cross section by about $30-40 \%$ is predicted and for $p_{m}=0.4 \mathrm{GeV} / c$ and $p_{m}=0.5 \mathrm{GeV} / c$ an increase of the cross section by more than a factor of two is predicted. The location of the extremum of the rescattering contributions give additional information about the details of the rescattering process such as the importance of the Fermi motion of the bound nucleons.

In order to address these questions in Hall $\mathrm{A}$, the $\mathrm{D}\left(\mathrm{e}, \mathrm{e}^{\prime} \mathrm{p}\right) \mathrm{n}$ cross section has been measured for $Q^{2}=$ $0.8,2.1$ and $3.5(\mathrm{GeV} / c)^{2}$ and missing momenta between $p_{m}=0$ and $p_{m}=0.5 \mathrm{GeV} / c$. A very preliminary result is shown in fig. 8 where the observed yield is compared to a Monte Carlo calculation using the PWIA for the cross section. Clearly the predicted angular dependence has been observed. A detailed analysis is currently in progress. 


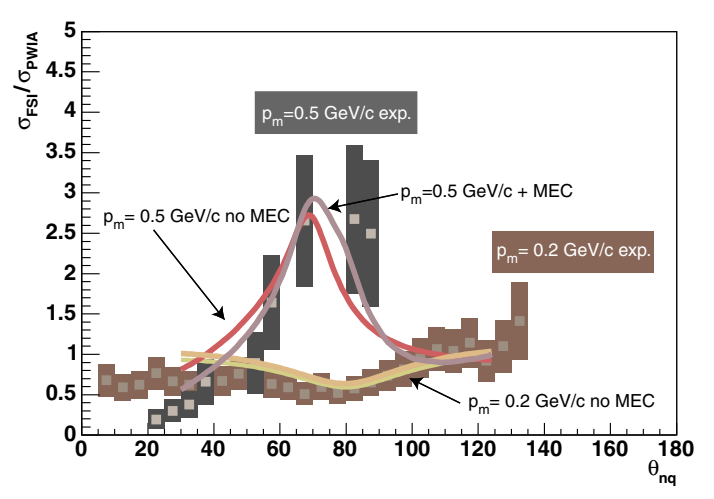

Fig. 8. Preliminary ratio of the measured yield to the calculated one using PWIA for the $\mathrm{D}\left(\mathrm{e}, \mathrm{e}^{\prime} \mathrm{p}\right) \mathrm{n}$ reaction at $Q^{2}=$ $3.5(\mathrm{GeV} / c)^{2}$. Data for $p_{m}=0.5(\mathrm{GeV} / c)$ show a strong enhancement of the cross section at about $70^{\circ}$ while the data for $p_{m}=0.2(\mathrm{GeV} / c)$ show a reduction at a similar angle. The bars indicate the uncertainty from the preliminary status of the analysis. The final errors will be of the order of $10 \%$. The calculation by J. M. Laget [32] reproduces the trend of the data quite well down to angles of about $40^{\circ}$. The behavior of the data at angles below $40^{\circ}$ is unexpected but given the status if the analysis no further conclusions can be drawn at this time.

\section{$4{ }^{3} \mathrm{He}$ and ${ }^{4} \mathrm{He}$ studies}

The breakup of the ${ }^{3,4} \mathrm{He}$ nuclei can lead to a 2-body final state like in the deuteron or it can lead to a 3-and even 4-body final state for ${ }^{3} \mathrm{He}$ and ${ }^{4} \mathrm{He}$, respectively. Only recently, with the advent of high computing power and efficient computational techniques, can the continuum final state be calculated accurately. These nuclei are the simplest systems in which to study short range correlations.

\subsection{Low $Q^{2}$ experiments}

Similar to deuterium, early (e, e'p) experiments on ${ }^{3} \mathrm{He}$ and ${ }^{4} \mathrm{He}$ explored the cross section with the goal to obtain information on the momentum distribution [33,34]. The same problems as described in section 3.1 are encountered here. In addition to examining the momentum distribution one has also studied the missing-energy spectrum. Early experiments on ${ }^{3} \mathrm{He}$ by Marchand et al. [33] showed a structure in the missing energy spectrum that is shifting with increasing recoil momentum in agreement with the kinematics of scattering off a nucleon pair. After the absorption of the virtual photon by one member of the pair, the struck nucleon is observed and the other partner of the pair recoils with the negative initial momentum of the struck nucleon.

To investigate the nucleon knock-out reaction in detail on these nuclei, a high-precision measurement of the (e, $\left.\mathrm{e}^{\prime} \mathrm{p}\right)$ cross section for ${ }^{3,4} \mathrm{He}$ has been carried out at MAMI. The goal was to determine the longitudinal and the transverse response in parallel kinematics close to the quasi-free peak and for missing energies up to $70 \mathrm{MeV} / c$.

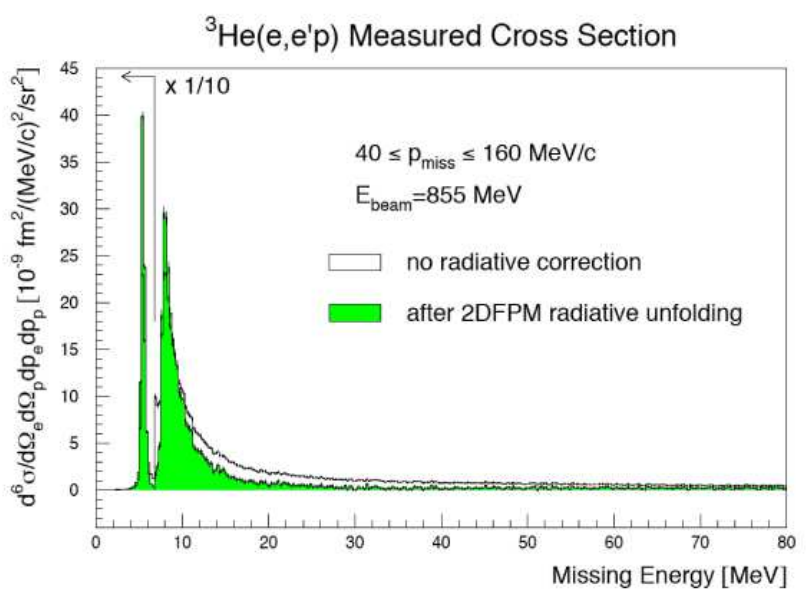

Fig. 9. The missing energy spectrum for the ${ }^{3} \mathrm{He}\left(\mathrm{e}, \mathrm{e}^{\prime} \mathrm{p}\right)$ reaction on the quasi-free peak. The 2-body breakup peak is clearly separated from the 3-body continuum. All strength above about $25 \mathrm{MeV}$ is entirely due to the radiative tail [38].
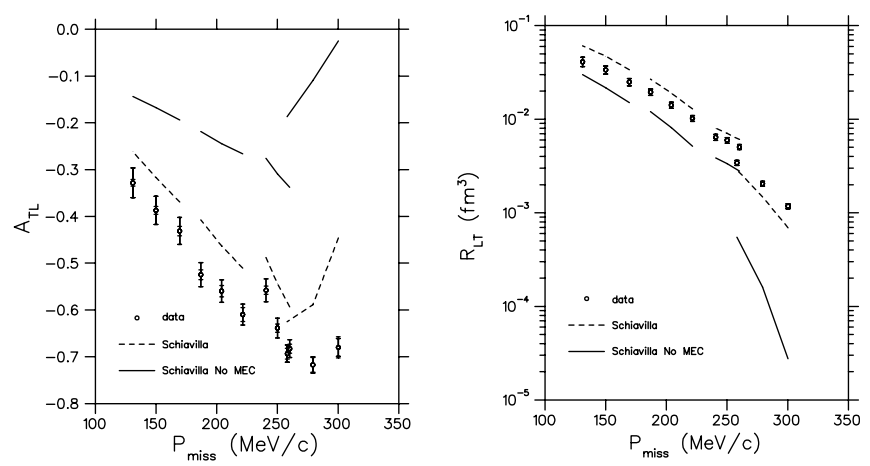

Fig. 10. The asymmetry $A_{L T}$ and the response function $R_{L T}$ measured at MAMI for the ${ }^{4} \mathrm{He}\left(\mathrm{e}, \mathrm{e}^{\prime} \mathrm{p}\right)^{3} \mathrm{H}$ reaction [39] together with calculations with and without the inclusion of MEC [40].

Results of these measurements can be found in references $[35,36]$. As an example, fig. 9 shows the missing energy spectrum obtained on the quasi-free peak. No additional strength can be observed above a missing energy of about $25 \mathrm{MeV}$ and the dependence of the cross section on the polarization of the virtual photon is the same as the one within PWIA. Hence, besides an overall reduction of the experimental cross section that is most likely due to FSI, no additional effects have been observed. By contrast, an $R_{L} / R_{T}$ separation carried out at Saclay [37] for missing momenta above $250 \mathrm{MeV} / c$ found very large deviations of the data from the calculations for the 2-body breakup, even when FSI and MEC are included.

Cross sections have also been obtained for the 3body breakup region. Again no additional dependence on the virtual photon polarization has been found beyond PWIA [36].

For the 2-body breakup of ${ }^{4} \mathrm{He}$ the interference response function, $R_{L T}$ has been extracted at MAMI [39] for missing momenta from $150 \mathrm{MeV} / c$ up to $300 \mathrm{MeV} / c$ at a momentum transfer $Q^{2} \approx 0.33(\mathrm{GeV} / c)^{2}$. The results 

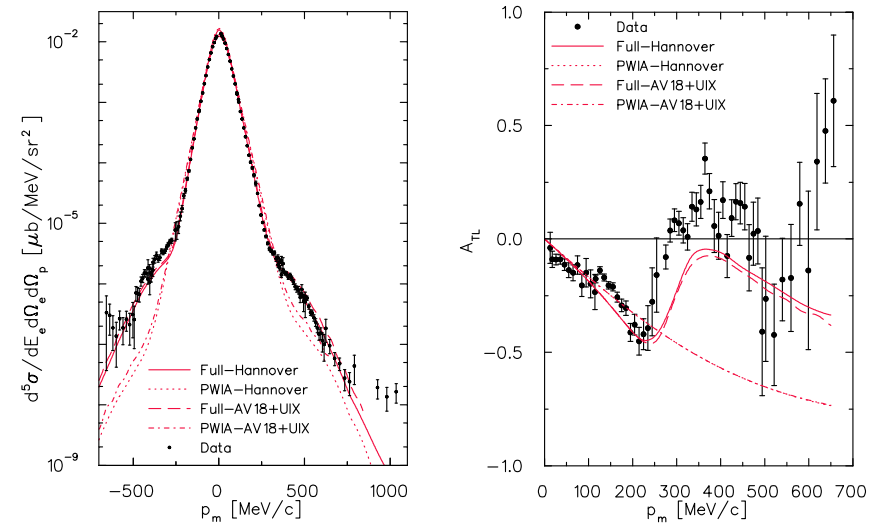

Fig. 11. Left: The cross section for the 2-body breakup of ${ }^{3} \mathrm{He}$ for $Q^{2}=1.55(\mathrm{GeV} / c)^{2}[42]\left(p_{m}<0\right.$ corresponds to $\phi=0^{\circ}$ and $p_{m}>0$ corresponds to $\phi=180^{\circ}$ ) Right: the extracted $A_{L T}$ ratio. The calculations are Glauber-based by J.M. Laget (see [42]).

have been compared to calculations by R. Schiavilla et al. [40,41], which show the need for MEC to improve the agreement with the experiment (fig. 10).

\subsection{High- $Q^{2}$ experiments}

A detailed study of the ${ }^{3,4} \mathrm{He}$ electrodisintegration in quasi-free kinematics has been performed in Hall A at Jefferson Lab. The (e, $\left.e^{\prime} p\right)$ cross section has been measured in parallel kinematics to allow for a $R_{L} / R_{T}$ separation and in perpendicular kinematics in order to determine the $A_{L T}$ asymmetry. The energy and 3-momentum transfers have been kept constant at $\omega=0.84(\mathrm{GeV})$ and at $q=1.502(\mathrm{GeV} / c)\left(Q^{2}=1.55(\mathrm{GeV} / c)^{2}\right)$ and cross sections have been measured up to $p_{m}=1 \mathrm{GeV} / c$. At these large momentum and energy transfers Glauber based calculations are expected to be valid.

In fig. 11 the experimental cross sections for the 2-body breakup are shown. Negative values of $p_{m}$ correspond to cross sections measured at $\phi=0^{\circ}$ while positive $p_{m}$-values are cross sections measured at $\phi=180^{\circ}$. Full Glauberbased calculations with modern 3-body wave functions from realistic potentials provide an excellent description of the experimental data up to missing momenta of $150 \mathrm{MeV} / c$. The same calculations give a good description of the observed cross sections up to missing momenta of $750 \mathrm{MeV} / c$. Some deviations between experiment and the calculation can be observed for $\phi=0^{\circ}$ between 250 and $500 \mathrm{MeV} / c$. It is also evident that FSI play a major role for recoil momenta above $300 \mathrm{MeV} / c$. In Laget's calculation MEC and IC are found to contribute at most $\approx 25 \%$ which is in agreement with the expectation that these contributions diminish with increasing momentum transfer.

Another recent Glauber-based calculation by R. Schiavilla et al. [43], where the full spin and isospin dependence of the underlying NN amplitudes is retained, reproduces the experimental 2-body breakup cross section very well (fig. 12). Rescattering effects have been found to be important over the full range of recoil momenta studied and,
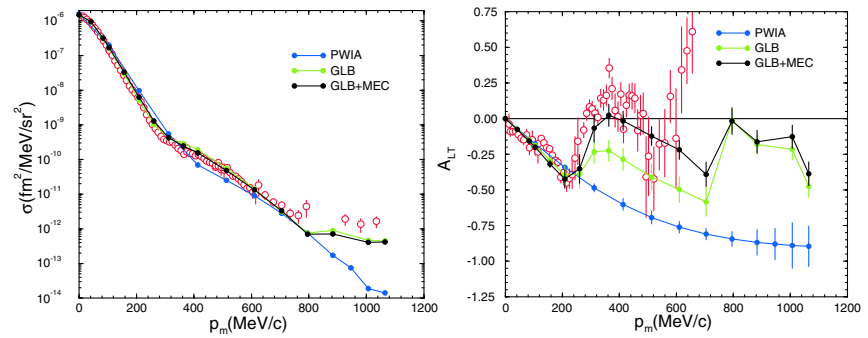

Fig. 12. As in fig. 9 but calculations by Schiavilla et al. [43] MEC contribute very little to the cross section while a sizeable contribution to $A_{L T}$ for $300 \leq p_{m}$ is observed. At missing momenta above $800(\mathrm{MeV} / c)$ double rescattering dominates the cross section.

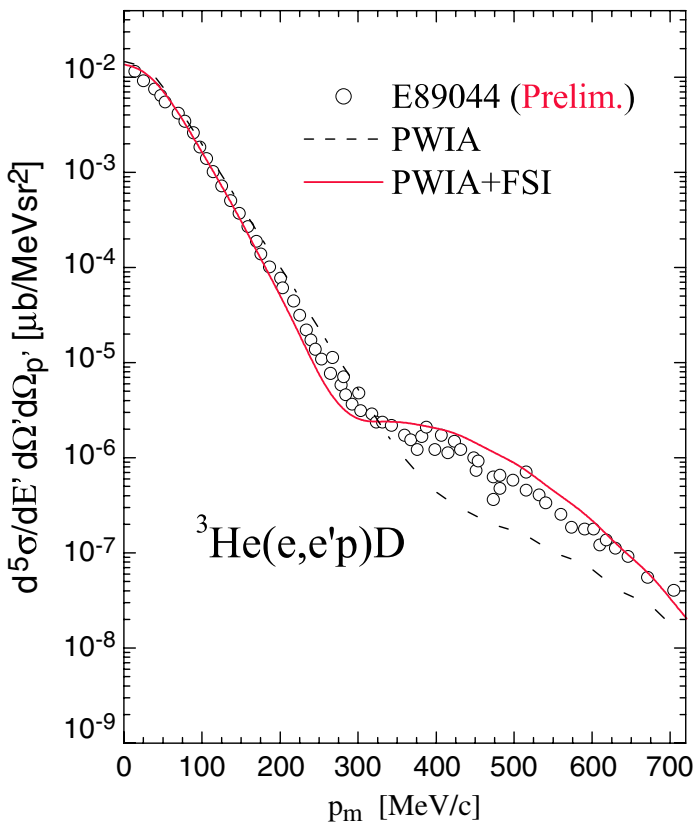

Fig. 13. The cross section for the 2-body breakup of ${ }^{3} \mathrm{He}$ for $Q^{2}=1.55(\mathrm{GeV} / c)^{2}[42]$ compared to a calculation by Kaptari et al. [44].

especially, double rescattering is important to improve the agreement between experiment and theory for recoil momenta above $750 \mathrm{MeV} / c$.

A third calculation by Kaptari et al. [44], based on GEA, can also reproduce the measured cross sections up to $p_{m}=700 \mathrm{MeV} / c$ reasonably well. In this calculation MEC and IC contributions have not been included (fig. 13). This again supports the expectation that with increasing momentum transfer, MEC and IC contributions to the cross section decrease.

In addition to 2-body breakup, the 3-body breakup reaction has also been observed at missing energies up to a $140 \mathrm{MeV}$ [45]. As in the Saclay experiment at low momentum transfer, a broad bump has been observed whose location shifts to increasing missing energy with increasing missing momentum (fig. 14). The peak location is kinematically in agreement with the breakup of a nucleon pair. The observed nucleon momentum is the sum of the momentum transfer and the initial momentum in the pair and 


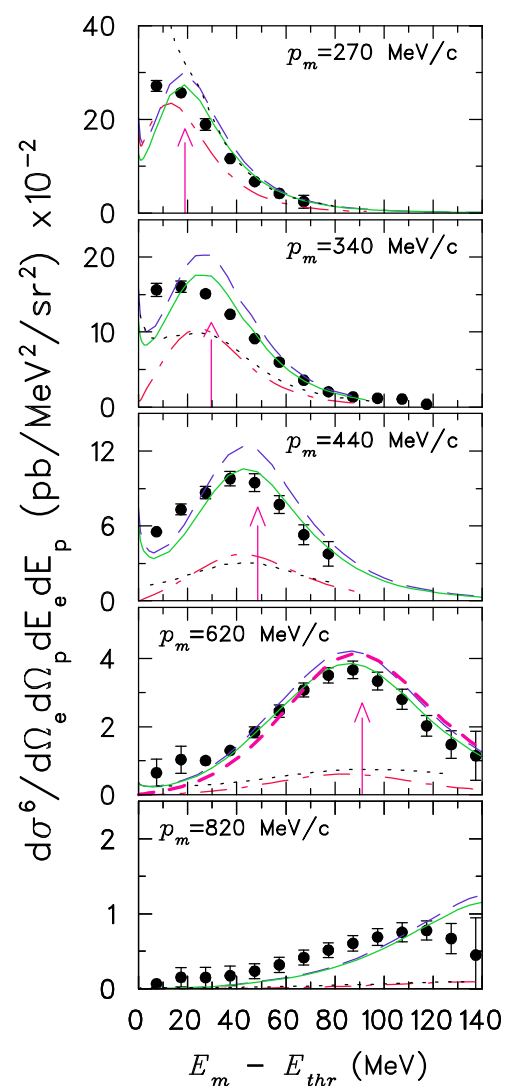

Fig. 14. Missing energy spectra for the 3-body beakup of ${ }^{3} \mathrm{He}$ in Hall $\mathrm{A}$ at JLAB [45], the kinematics is the same as in reference [42]. The arrows indicate the expected location of the peak for the breakup of a nucleon pair. The width is a consequence of the center-of-mass motion of the pair. The solid line includes FSI and MEC. The calculations are by J.M. Laget (see [45]).

the recoiling nucleon is the partner. The third nucleon in helium is a spectator.

Calculations by J. M. Laget [46] show that FSI within the active nucleon pair contribute strongly to the observed structure while final state interactions between the pair nucleons and the spectator nucleon seem to be much less important. Integrating over missing energy and dividing by the electron-nucleon cross section [8] results in the (PWIA) effective momentum distribution for the 3-body breakup. This can then be compared to the effective momentum distribution from the 2-body breakup channel. The result is shown in fig. 15 . With increasing recoil momenta the momentum distribution is dominated by the 3-body breakup process. However at this point one cannot experimentally distinguish contributions due to FSI within the nucleon pair from contributions of shortrange correlations. Maybe separated response functions will give more information to address this question.

\subsection{Search for short-range correlations in ${ }^{3} \mathrm{He}$}

The detection of nucleon pairs should allow one to study nucleon correlations in a direct way. These triple-

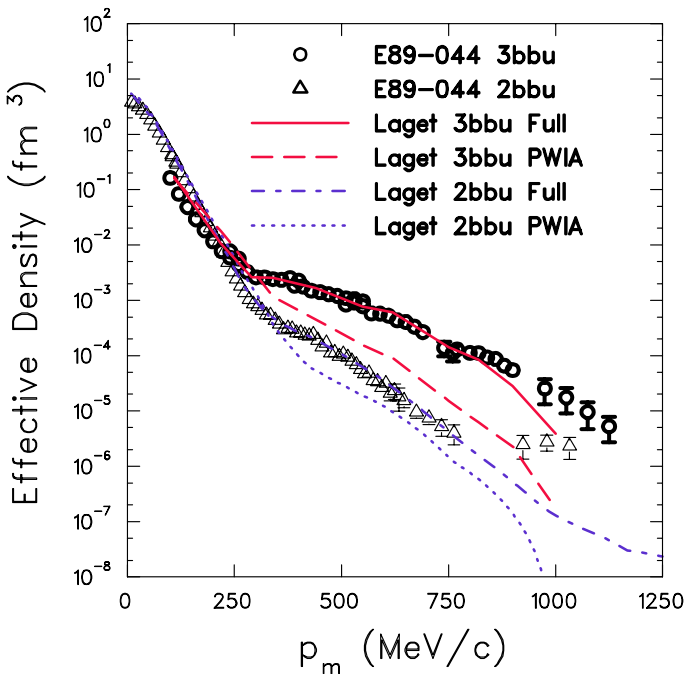

Fig. 15. Proton effective momentum distributions in ${ }^{3} \mathrm{He}$ extracted from the 3-body breakup reaction (open circles) compared to the 2-body contribution (triangles) [45]. The integration in missing energy ranges from threshold up to $140 \mathrm{MeV}$. Calculations are by J. M. Laget $[46,32]$.

coincidence experiments can only be carried out with CW electron beams. ${ }^{3} \mathrm{He}\left(\mathrm{e}, \mathrm{e}^{\prime} \mathrm{NN}\right)$ experiments have been carried out at NIKHEF with the pulse stretcher ring AmPS, at MAMI and at Jefferson Lab. Again, one of the fundamental problems is to isolate the various reaction processes that can lead to the emission of nucleon pairs in addition to initial state correlations. The most prominent processes are final state interactions and 2-body currents such as MEC and IC. In an experiment carried out at NIKHEF $[47,48],{ }^{3} \mathrm{He}\left(\mathrm{e}, \mathrm{e}^{\prime} \mathrm{pp}\right) \mathrm{n}$ cross sections were measured for momentum transfers around $400 \mathrm{MeV} / c$ and at an energy transfer of about $220 \mathrm{MeV}$. The experimental results were compared to continuum Faddeev calculations performed with various modern potentials including oneand two-body currents. The relative momenta of pair nucleons was between 500 and $800 \mathrm{MeV} / c$. It was found that calculations performed with only a one-body hadronic current operator show a fair agreement with the data for small missing (neutron) momenta. This can be interpreted as a direct knock-out of a proton pair. With increasing missing momentum, contributions from MEC and IC also increase. FSI between the pair nucleons depend on the relative emission angle. Decreasing relative emission angle leads to increasing FSI.

At MAMI ${ }^{3} \mathrm{He}\left(\mathrm{e}, \mathrm{e}^{\prime} \mathrm{pn}\right) \mathrm{p}$ cross sections have been measured and are currently being analyzed.

Another study of the ${ }^{3} \mathrm{He}\left(\mathrm{e}, \mathrm{e}^{\prime} \mathrm{pp}\right) \mathrm{n}$ reaction has been carried out with CLAS at Jefferson Lab [49]. Via a Dalizplot of the kinetic energies of the observed nucleons divided by the energy transfer, various reaction mechanisms could be selected. The location where both nucleons have the smallest energy fraction corresponds to the process where the virtual photon is absorbed by the uncorrelated nucleon. The observed (small energy fraction) nucleons are then the pair nucleons. This interpretation is supported by 

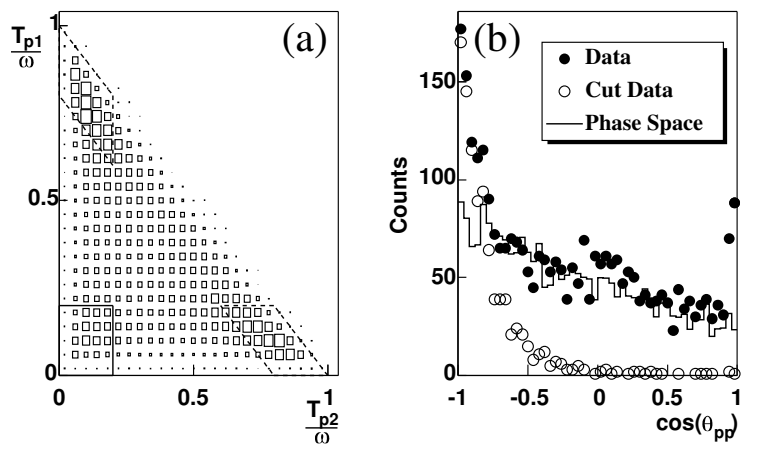

Fig. 16. a) Dalitz plot for the lab frame $T_{p 1} / \omega$ versus $T_{p 2} / \omega$ for events with $p_{N}>0.25 \mathrm{GeV} / c$ [49]. b) The cosine of the $p$ - $p$ lab frame opening angle. Open circles for events with small proton momenta $\left(T_{p}<0.2 \cdot \omega\right)$ and closed circles show all data.

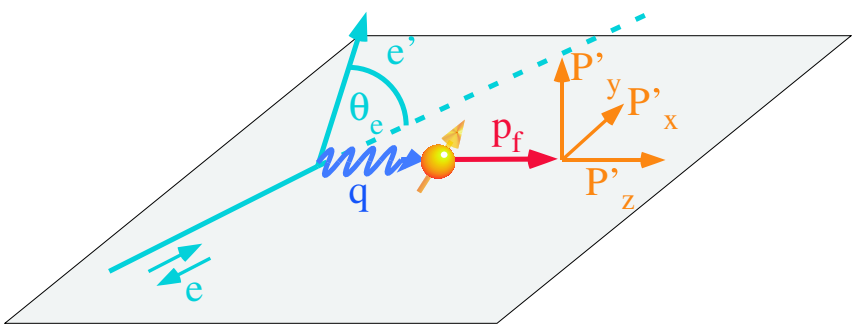

Fig. 17. Definition of polarization variables for $\left(e, e^{\prime} N\right)$ polarization transfer experiments. $e$ represents the polarized electron beam, $e^{\prime}$ the scattered electron and $\theta_{e}$ the electron scattering angle. $\boldsymbol{q}$ the virtual photon, $p_{f}$ the final nucleon momentum and $P_{x}^{\prime}, P_{y}^{\prime}, P_{z}^{\prime}$ the polarization of the ejected nucleon.

the relative angular distribution of the observed nucleon pair that shows a pronounced peak at $180^{\circ}$ (in the Lab frame) corresponding to nucleon emission back-to-back (fig. 16). As before $[47,48]$ one finds strong FSI within the nucleon pair and only small contributions due to twobody currents and rescattering of the struck nucleon with the pair.

\section{Polarization transfer experiments}

Spin degrees of freedom open up a new, large set of observables that make it possible to address a variety of different questions in nuclear physics. Spin observables lead to interference terms between different reaction amplitudes. This in turn makes it possible to study very small amplitudes when their effect is enhanced by a large one. An important application of this is the determination of the nucleon form factors where polarized electrons are scattered off an unpolarized target and the polarization of the struck nucleon is determined in a polarimeter [50].

For the $\left(\vec{e}, \mathrm{e}^{\prime} \vec{N}\right)$ reaction on a free nucleon one obtains (see fig. 17)

$$
\frac{G_{E}}{G_{M}}=-\frac{P_{x}^{\prime}}{P_{z}^{\prime}} \cdot \frac{\left(E_{i}+E_{f}\right)}{2 M} \tan \theta_{e} / 2,
$$

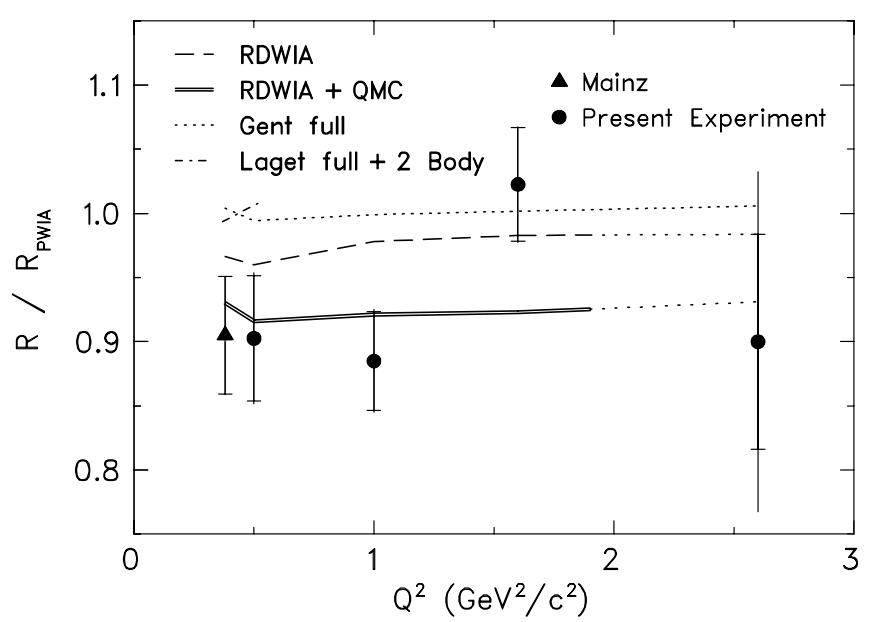

Fig. 18. Polarization transfer experiments on ${ }^{4} \mathrm{He}$ from Mainz [55] and JLAB [56]. The best agreement is obtained for a modified nucleon form factor within the quark-meson coupling model $[57,58]$.

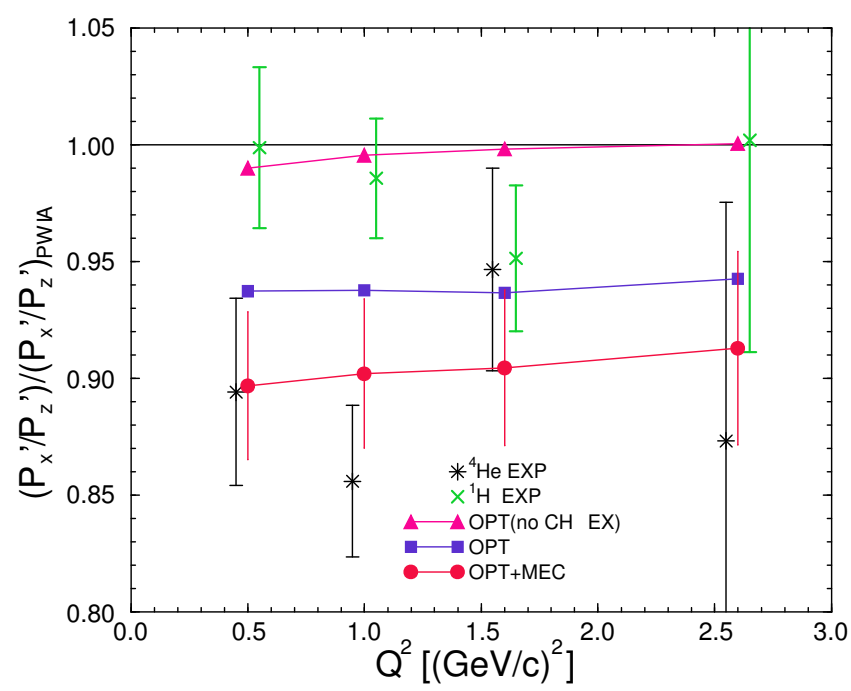

Fig. 19. Comparison of the ${ }^{4}$ He polarization transfer experiment [56] and the calculation by Schiavilla et al. [59]. No nucleon form factor modifications have been included. OPT: Only one-body currents included and $p^{3} H$ FSI described by an optical potential with and without (no CH-EX) charge exchange. OPT+MEC: Full optical potential including one- and two-body currents. The error bars in the calculation are due to the Monte Carlo method used and are similar for all calculations shown.

where $G_{E}$ and $G_{M}$ are the electric and magnetic Sachs form factors, $P_{x}^{\prime}$ and $P_{z}^{\prime}$ are the nucleon polarization, $E_{i}$ and $E_{f}$ the incident and scattered (or final) electron energy, $M$ the nucleon mass and $\theta_{e}$ the electron scattering angle.

This method has been used extensively to determine the neutron form factor and the high- $Q^{2}$ behavior of the ratio of the electric to the magnetic form factor of the proton $[51,52,53,54]$ (see contributions by D. Rohe and M. Ostrick). The same process can also be measured in nuclei. Experiments carried out at MAMI [55] and at 
JLAB [56] on ${ }^{4} \mathrm{He}$ showed that the ratio

$$
R=\frac{\left(P_{x}^{\prime} / P_{z}^{\prime}\right)_{H e}}{\left(P_{x}^{\prime} / P_{z}^{\prime}\right)_{H}}
$$

is reduced by about 10\% (fig. 18). Some calculations based on the quark meson coupling model $[57,58]$ suggest a possible modification of the nucleon form factors inside nuclei to account for this observation. Another calculation by Schiavilla et al. [59] uses realistic wave functions for the bound state that include correlation effects. One- and twobody currents are included and spin and isospin dependences in the final state interaction including charge exchange have been taken into account. The calculation can reproduce the experimental data without the need of form factor modifications as can be seen in fig. 19. New highprecision experiments on ${ }^{4} \mathrm{He}$ are planned at Jefferson Lab in Hall A to improve and extend the available data.

\section{Summary and conclusion}

In the last 20 years much progress has been made in the knowledge of the structure of few-body systems. The availability of high quality CW beams made it possible to measure coincidence cross sections over a wide range of kinematical variables which were inaccessible before. In parallel, theoretical progress together with increasing computational power has resulted in sophisticated models that agree very well with the data.

Coincidence data on ${ }^{3} \mathrm{He}$ have enjoyed a lot of attention and the analysis of recent data taken at Jefferson Lab is still in progress. The importance of a detailed understanding of final state interactions, MEC, and IC is crucial in order to extract information on the short-range structure of light nuclei.

While many new single-arm data on the deuteron have been obtained, available coincidence data and especially the lack of new, high-precision response function determinations are disappointing.

Triple coincidence experiments on the He nuclei are expected to provide new data on the structure of correlations. However, these experiments are very complex to carry out, analyze, and interpret. Several experiments are being currently analyzed at MAMI and JLAB. The (upgraded ) Mainz Microtron will continue to play a leading role in nuclear physics.

I would like to thank H. Arenhövel, H. Backe, D. Drechsel, J. Friedrich, K-H. Kaiser and Th. Walcher for making MAMI such a success, for their contributions to our field and for giving me the opportunity to carry out research at MAMI. I wish you all the best in the future. This work was supported in part by the Department of Energy, DOE grant DE-FG02-99ER41065.

\section{References}

1. A. Nogga, H. Kamada, W. Glöckle, Nucl. Phys. A 689, 357 (2001).
2. R. Schiavilla, V.R. Pandharipande, R.B. Wiringa, Nucl. Phys. A 449, 219 (1986).

3. J. Carlson, Phys. Rev. C 36, 2026 (1987).

4. B.S. Pudliner et al., Phys. Rev. Lett. 74, 4396 (1995).

5. A.E.L. Dieperink, T. de Forest, Annu. Rev. Nucl. Sci. 25, 1 (1975).

6. S. Frullani, J. Mougey, Adv. Nucl. Phys. 14, 1 (1984).

7. S. Boffi, C. Giusti, F.D. Pacati, Phys. Rep. 226, 1 (1993).

8. T. de Forest, Nucl. Phys. A 392, 232 (1983).

9. M. Bernheim et al., Nucl. Phys. A 365, 349 (1981).

10. S. Turck-Chieze et al., Phys. Lett. B 142, 145 (1984).

11. K.I. Blomqvist et al., Phys. Lett. B 429, 33 (1998).

12. P.E. Ulmer et al., Phys. Rev. Lett. 89, 062301-1 (2002).

13. H. Arenhövel, W. Leidemann, L. Tomusiak, Phys. Rev. C 52, 1232 (1995).

14. J.E. Ducret et al., Phys. Rev. C 49, 1783 (1994).

15. D. Jordan et al., Phys. Rev. Lett. 76, 1579 (1996).

16. Kasdorp et al., Few-Body Syst. 25, 115 (1997).

17. M. van der Schaar et al., Phys. Rev. Lett. 66, 2855 (1991).

18. M. van der Schaar et al., Phys. Rev. Lett. 68, 776 (1992).

19. W. Fabian, H. Arenhövel, Nucl. Phys. A 314, 253 (1979).

20. H. Arenhövel, private communication (2001).

21. R. Boehm, Thesis, University of Mainz (2001).

22. S. Jeschonnek, J.W. Van Orden, Phys. Rev. C 62, 044613 (2000).

23. W.U. Boeglin, private communication (2005).

24. Z.-L. Zhou et al., Phys. Rev. Lett. 87, 172301 (2001).

25. Z.-L. Zhou et al., Proceedings of the MIT-Bates Workshop (1998).

26. F. Ritz, H. Göller, Th. Wilbois, H. Arenhövel, Phys. Rev. C 52, 1232 (1995).

27. E. Hummel, J.A. Tjon, Phys. Rev. C 49, 21 (1994).

28. A. Pellegrino et al., Phys. Rev. Lett. 78, 4011 (1997).

29. S.M. Dolfini et al., Phys. Rev. C 60, 064622 (1999).

30. H.J. Bulten et al., Phys. Rev. Lett. 74, 4775 (1995).

31. L.L. Frankfurt, M.M. Sargsian, M.I. Strikman, Phys. Rev. C 56, 1124 (1997).

32. J.M. Laget, Phys. Lett. B 609, 49 (2005).

33. C. Marchand et al., Phys. Rev. Lett. 60, 1703 (1988).

34. E. Jans et al., Nucl. Phys. A 475, 687 (1987).

35. R.E.J. Florizone et al., Phys. Rev. Lett. 83, 2308 (1999).

36. A. Kozlov et al., Phys. Rev. Lett. 93, 132301 (2004).

37. J.M. Le Goff et al., Phys. Rev. C 55, 1600 (1997).

38. R.E.J. Florizone, Thesis, MIT (1999).

39. K. Aniol et al., Eur. Phys. J. A 22, 449 (2004).

40. R. Schiavilla et al., Phys. Rev. Lett. 54, 835 (1990).

41. J. Forest et al., Phys. Rev. C 54, 646 (1996).

42. M. Rvachev et al., Phys. Rev. Lett. 94, 192302 (2005).

43. R. Schiavilla et al., Phys. Rev. C 72, 064003 (2005).

44. L.P. Kaptari, C. Ciofi degli Atti, Phys. Rev. C 71, 024005 (2005).

45. F. Benmokhtar et al., Phys. Rev. Lett. 94, 082305 (2005).

46. J.M. Laget, Few-Body Syst., Suppl. 15, 171 (2003).

47. D.L. Groep et al., Phys. Rev. Lett. 83, 5443 (1999).

48. D.L. Groep et al., Phys. Rev. C 63, 014005 (2000).

49. R.A. Niyazov et al., Phys. Rev. Lett. 92, 52303 (2004).

50. R. Arnold et al., Phys. Rev. C 23, 363 (1981).

51. M.K. Jones et al., Phys. Rev. Lett. 84, 1389 (2000).

52. O. Gayou et al., Phys. Rev. C 64, 038202 (2001).

53. O. Gayou et al., Phys. Rev. Lett. 88, 092301 (2002).

54. T. Pospischil et al., Eur. Phys. J. A 12, 125 (2001).

55. S. Dietrich et al., Phys. Lett. B 500, 47 (2001).

56. S. Strauch et al., Phys. Rev. Lett. 91, 052301 (2003).

57. D.H. Lu et al., Phys. Lett. B 417, 217 (1998).

58. D.H. Lu et al., Phys. Rev. C 60, 068201 (1999).

59. R. Schiavilla et al., Phys. Rev. Lett. 94, 072303 (2005). 